\title{
Root Raised Cosine Pulse Shape Based ICI Suppression in OFDM System for Rayleigh Multipath Channel
}

\author{
Ashok Khedkar $^{\# 1}$, M.Sumathi ${ }^{\$ 2}$, M.Murugan ${ }^{* 3}$ \\ \#Department of Electronics \& Telecommunication, Cummins College of Engineering for Women Pune-411052, \\ Maharashtra \& Research Scholar, Sathyabama University, Chennai-600119, Tamil Nadu, India. \\ 1ashok.khedkar81@gmail.com \\ ${ }^{\$}$ Department of Electronics \& Communication Engineering, Sathyabama University, Chennai-600119, TN, \\ India. \\ *Department of Electronics \& Communication Engineering, Valliammai Engineering College, Chennai-603203, \\ TN, India. \\ ${ }^{2}$ sumagopi206@gmail.com \\ 3r.murugan.m@gmail.com
}

\begin{abstract}
Orthogonal frequency division multiplexing is one of the potential scheme used in broadband communication systems to support high speed data transmission capability with excellent performance in multipath fading channel. The major benefit of OFDM scheme lies in its capability to convert frequency selective fading channel into flat faded channel, and its large spectral efficiency. However one of the negative aspect of OFDM system is its sensitive nature to the frequency offset present amongst the subcarriers. This Carrier frequency offset introduces Inter Carrier Interference (ICI), and this redundant ICI degrade the system performance. In this paper, first the authors have analyzed the error performance of QPSK modulated OFDM system in the existence of carrier frequency offset for Rayleigh multipath channel. Then Inter Carrier Interference power (ICI) and Carrier to Interference ratio (C/I) is analyzed for varying frequency offset. The authors have examined the increasing nature of Inter Carrier Interference (ICI) power and decreasing nature of Carrier to Interference ratio for varying frequency offset. Root Raised Cosine pulse shape based technique is proposed for the reduction of ICI and optimum value of roll-off factor is obtained for the minimum value of ICI.
\end{abstract}

Keywords- OFDM, Doppler shift, RRC Filter ICI, CIR, Rayleigh channel

\section{INTRODUCTION}

The principle of OFDM is similar to the multi-carrier transmission (MCT) technique, which provides reduction in ISI and less bandwidth per subcarrier, thereby allowing high data rate and simple equalization in multipath channel [1]. OFDM can be implemented using FFT and IFFT with digital baseband modulation schemes such as QPSK, and QAM. The baseband OFDM symbol at the transmitter output is represented by

$x(n)=\sum_{k=0}^{N-1} X[k] e^{j 2 \pi k n / N} ; n=0 \ldots . N-1$

Where $\mathrm{X}[\mathrm{k}]$ represents complex symbol transmitted over $\mathrm{k}^{\text {th }}$ sub carrier and $\mathrm{N}$ is the number of subcarriers used in the system. Figure (1) shows the basic blocks of the transmitter and receiver in an OFDM scheme. At transmitter, with the help of mapper block input information is mapped into complex symbols and transformed into a parallel form using serial to parallel block. Symbols obtained at the output of serial to parallel block are modulated over an individual subcarrier by IDFT block. The output of IDFT/IFFT block is first transformed into a serial form and with the help of DAC it is changed into analog form. In the receiver side first information is obtained in digital form using ADC and it is demodulated using DFT/FFT operation. The demodulated symbols are de mapped into original information. 


\section{CAUSES OF INTERCARRIER INTERFERENCE}

\section{A. Carrier frequency offset}

Frequency offset is typically caused due to the presence of small frequency disparity in the local oscillators of transceiver [9]. All OFDM subcarriers are orthogonal if the frequencies are multiples of fundamental frequency in the given FFT interval. In the existence of frequency offset, inter carrier interference (ICI) is introduced due to the loss of orthogonality between the subcarriers. This ICI corrupt the performance of the system, and it is inversely proportional to the subcarrier frequency spacing.

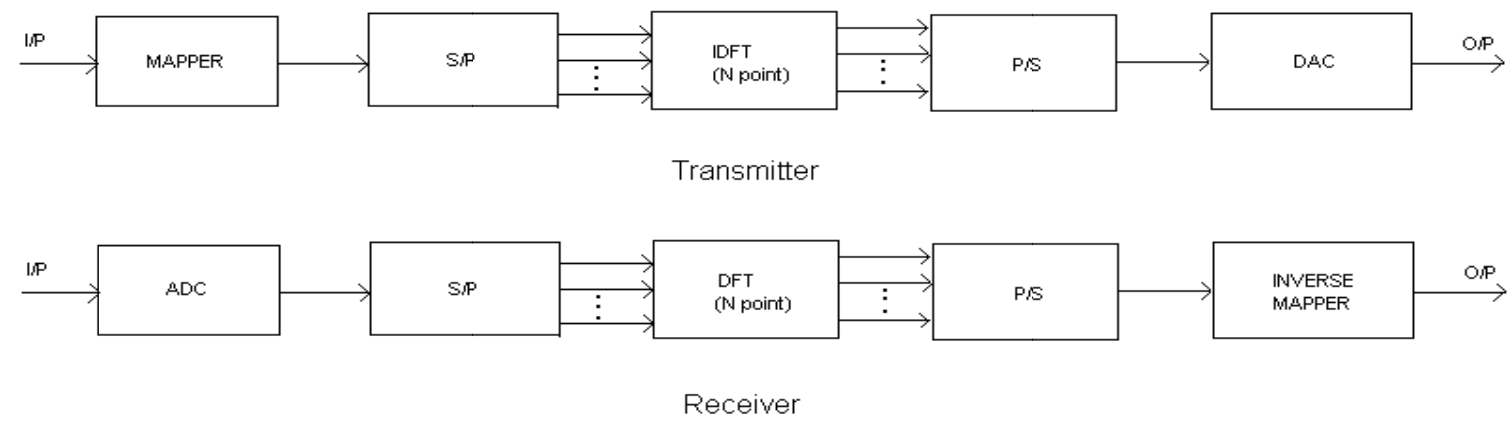

Fig. 1. Basic OFDM system

\section{B. Doppler shift}

Another reason for the introduction of ICI is time varying nature of channel caused by the relative movement in the transceiver [6]. Doppler shift can be neglected in indoor environments but it is dominant for outdoor type of communication systems. Doppler shift or change in frequency $\mathrm{f}_{\mathrm{d}}$ is given by $\mathrm{f}_{\mathrm{d}}=\frac{v}{\lambda} * \cos \theta$ [2], where $\mathrm{v}$ is the velocity of the receiver, $\lambda$ is the wavelength of transmitted signal, and $\theta$ represents spatial angle between the line joining the receiver with the incoming signal.

\section{ICI ANALYSIS}

The key drawback of OFDM is its susceptible nature to Carrier Frequency Offset (CFO). The impact of a frequency offset can be seen as an inaccuracy in the frequency instant, where the received signal is sampled in demodulator by the FFT. The presence of CFO in the OFDM system bring in Inter Carrier Interference (ICI) and leads to the reduction of Carrier to Interference ratio(C/I).

If $\Delta \mathrm{f}$ represents frequency offset, $\mathrm{B} / \mathrm{N}$ is the subcarrier bandwidth of an OFDM symbol then the normalized frequency offset $(\epsilon)$ is specified by [9]

$$
\epsilon=\Delta f /\left(\frac{B}{N}\right)
$$

An OFDM symbol at the output of FFT block, which is not affected by CFO, is of the following nature.

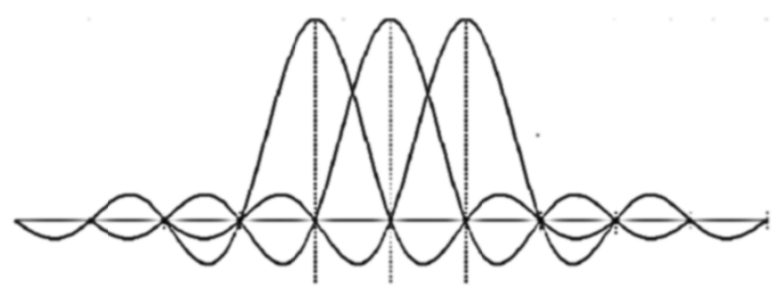

Fig. 2. OFDM symbol without carrier synchronization error.

In such a case, the peak of one subcarrier coincides with the null of all other subcarriers. Therefore at the sample time there is no interference from the adjacent subcarriers. The mathematical equation of such a symbol is specified by

$$
X[k]=\sum_{n=0}^{N-1} x(n) e^{-j 2 \pi k n / N} \quad ; \quad k=0 \ldots . N-1
$$

Where $\mathrm{N}$ represents the total number of subcarriers present within the system. The presence of CFO causes a shift in the subcarriers such that the peak of one no longer coincides with the null of adjacent subcarriers. Therefore at the sampling time there is interference from the adjacent subcarriers. The OFDM symbol affected by $\mathrm{CFO}$ is shown in figure 3 . 


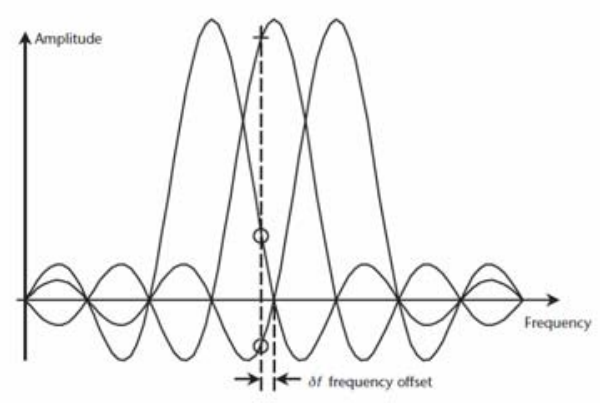

Fig. 3. Symbol affected by ICI

The mathematical equation of a CFO affected OFDM symbol is specified by

$$
X[k+\epsilon]=\sum_{n=0}^{N-1} x(n) e^{-j 2 \pi(k+\epsilon) n / N} ; k=0 \ldots . N-1
$$

The power of an OFDM symbol with $\mathrm{N}$ subcarriers is specified by

$P=\frac{\sum_{k} X[k] * X[k]^{*}}{N^{2}} \quad ; \quad k=0 \ldots . N-1$

The difference in the power of an OFDM symbol affected by CFO and the power of an OFDM symbol not affected by CFO is equal to the total ICI present in that symbol. Average ICI over the entire transmitted and received OFDM symbols gives the total ICI present in the system.

This paper suggests the use of Root raised cosine pulse shape based filter to reduce ICI. Further optimized value of roll off factor is obtained for minimum value of ICI.

\section{PULSE SHAPING}

Pulse shaping filters are used to confine the signal bandwidth, to enhance the data rate and to reduce transmission errors such as ISI (Inter Symbol Interference) [5][8]. Pulse shaping filter reduces the side lobes of individual subcarrier, which is the main cause of ICI, when the orthogonality of subcarriers get lost due to the carrier frequency offset. The two important properties of the perfect pulse shaping filter are, a large stop-band attenuation to diminish ICI and minimized ISI to attain low bit error rate (BER).

According to the Nyquist criteria, the impulse response of the shaping filter should have null crossings at multiples of the symbol duration to achieve ISI free transmission and to assist in timing recovery [5][8]. The filter that satisfies Nyquist criterion has a sinc pulse in the time domain with a brick wall frequency response. This type of filter is difficult to realize in practice and basically the impulse response of perfect continuous filter is sampled to obtain a realizable filter. For this the sampling rate should be double than the symbol rate of the message, i.e. the filter must interpolate the data by a minimum factor of two or more. The raised cosine filter satisfies Nyquist criteria and it is band-limited to $(1+\beta) \mathrm{R} / 2$, where $\beta$ is the roll-off factor and $\mathrm{R}$ is the data rate. Its impulse response is specified by $p(t)=\frac{\cos 2 \pi \beta t}{1-(4 \pi \beta t)^{2}}(\sin (\pi R t) / \pi R t)$ with zero crossings once every $\mathrm{T}_{\mathrm{b}}$ seconds.

\section{ROOT RAISED COSINE FILTER}

The raised cosine filtering operation can be split and implemented at the transmitter and receiver both. The transfer functions of these filters are such that their multiplication is equal to the transfer function of a single raised cosine pulse shaping filter. To optimize signal to noise ratio at the receiver, the transfer function of the raised cosine filter, $\mathrm{P}(\mathrm{f})$ is split equally between the transceiver, thus giving

$$
H t(f)=H r(f)=\sqrt{P(f)}
$$

This is called 'square root raised cosine filter' having impulse response of

$h(t)=h(r)=\frac{2 \beta}{\pi \sqrt{T}} \frac{\cos [(1+\beta) \pi t / T]+\frac{\sin [(1-\beta) \pi t / T]}{4 \beta t / T}}{1-(4 \beta t / T)^{2}}$

The pulse shaping filter at the transmitter performs up-sampling of the signal whereas, the matched filter at the receiver performs down-sampling. This approach provides better stop band attenuation than using a single raised cosine filter at the transmitter and a down-sampler at the receiver. It also reduces Inter symbol Interference (ISI) among the symbols. The matched filter detects a pulse with a known shape $\mathrm{p}(\mathrm{t})$ in the received symbol $\mathrm{Y}(\mathrm{t})$ which is of the form $\mathrm{p}(\mathrm{t})+\mathrm{N}(\mathrm{t})$. $\mathrm{N}(\mathrm{t})$ is additive, stationary noise with zero mean. 


\section{ADAPTIVE PULSE SHAPING}

The roll-off factor $(\beta)$ of the pulse shaping function determines the width of the transition band of the pulse. As $\beta$ increases from 0 to 1 , the pulse period decreases and also there is an increase in the side lobe attenuation. Thus the restriction of signal over time domain causes expansion in frequency domain and signal will consume excess bandwidth. So there must be a compromise between value of $\beta$ and the bandwidth in order to achieve undistorted transmission of a pulse over a narrowband channel. Realistic communication systems utilize a roll off factor between 0.1 and 0.5.In adaptive pulse shaping the authors vary the roll off factor from 0.1 to 0.5 and obtained the ICI power at these values. The value of $\beta$ which gives minimum ICI is the optimized roll off factor.

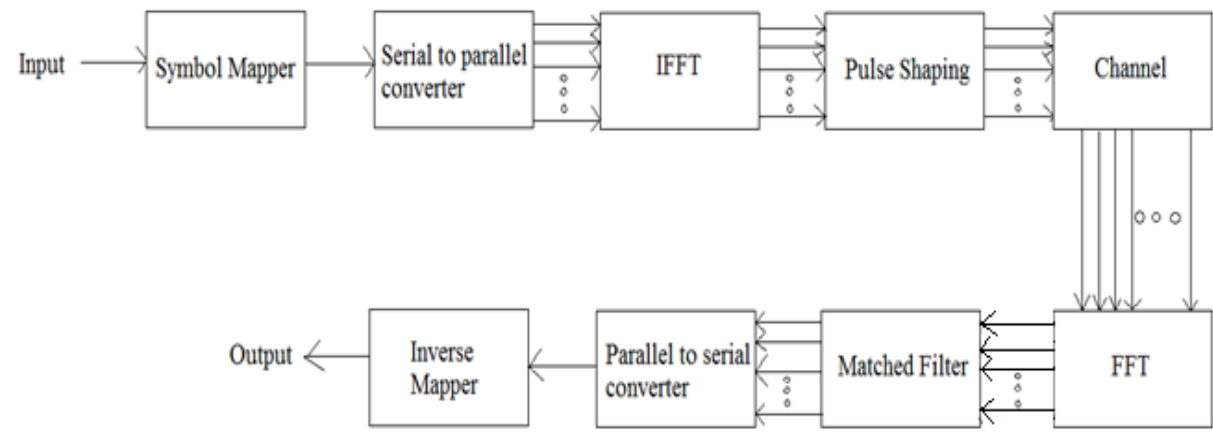

Fig. 4. Block diagram of implemented system

\section{SIMULATION RESULTS}

Implemented OFDM system for QPSK modulation scheme is shown in Figure 4. System is implemented for 64 subcarriers and channel is modelled as a Rayleigh multipath channel with 2 discrete paths. The time domain symbols available at the output of IDFT/IFFT block are given to the Root Raised Cosine (RRC) pulse shaping filter. The matched filter used at the receiver is also a Root Raised Cosine filter. From Figure 5 it is understood that error performance of QPSK modulated OFDM system gets degraded in the presence of frequency offset. Figures 6 and 7 compare ICI and C/I with respect to CFO for pulse shaped OFDM symbols with that of without shaped symbols in Rayleigh multipath channel. It is observed that at a value of 0.1 frequency offset the ICI for original system (without using pulse shaping) is approximately $-41 \mathrm{~dB}$ (close to $10^{-4}$ ) and after using RRC filtering it will be approximately $-47 \mathrm{~dB}$ (close to $10^{-5}$ ). Also $\mathrm{C} / \mathrm{I}$ ratio is enhanced from $4 \mathrm{~dB}$ (above $10^{\circ}$ ) to 10 $\mathrm{dB}$ (close to $10^{1}$ ). Thus due to the use of Root Raised Cosine filter ICI get reduced and C/I ratio gets improved. Figure 8 helps to obtain optimum roll off factor $(\beta)$ to achieve minimum ICI.

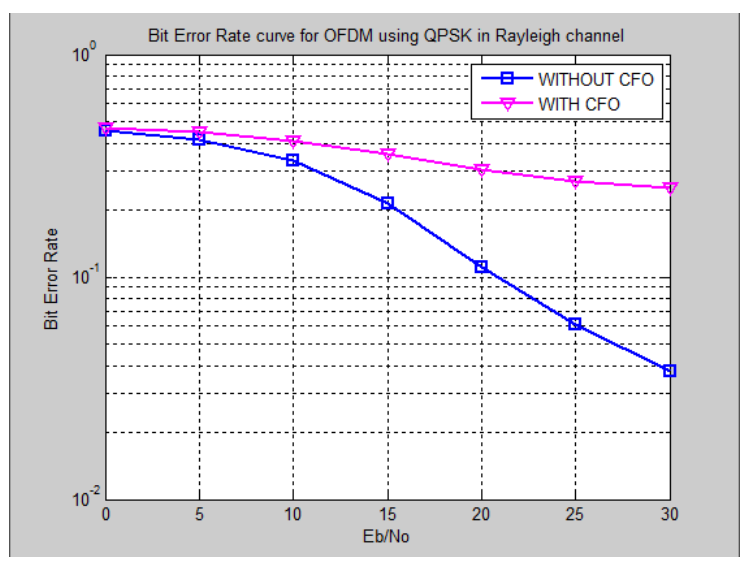

Fig. 5. Comparison of BER vs. Eb/No for an OFDM System affected by CFO and not affected by CFO in Rayleigh multipath channel

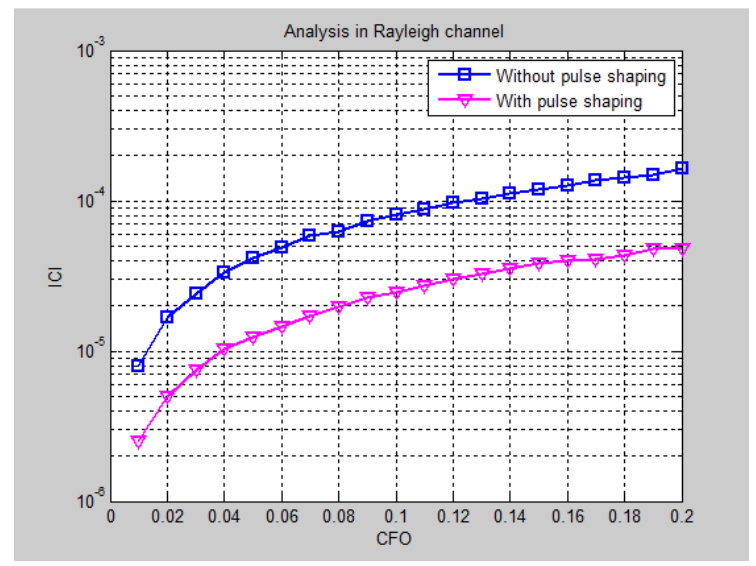

Fig. 6. Comparison of ICI vs. CFO for pulse shaped OFDM symbols with those without pulse shaping in Rayleigh multipath channel 


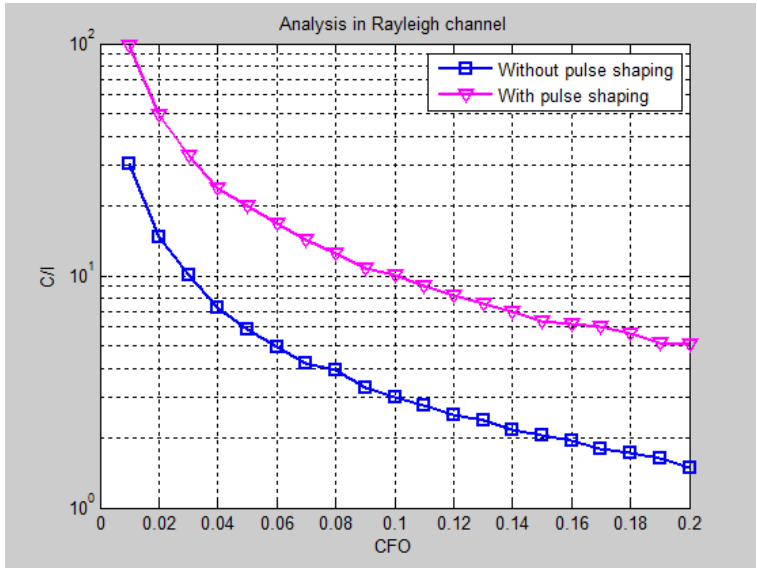

Fig. 7. Comparison of C/I vs. CFO for pulse shaped OFDM Symbols with those without pulse shaping in Rayleigh Multipath channel

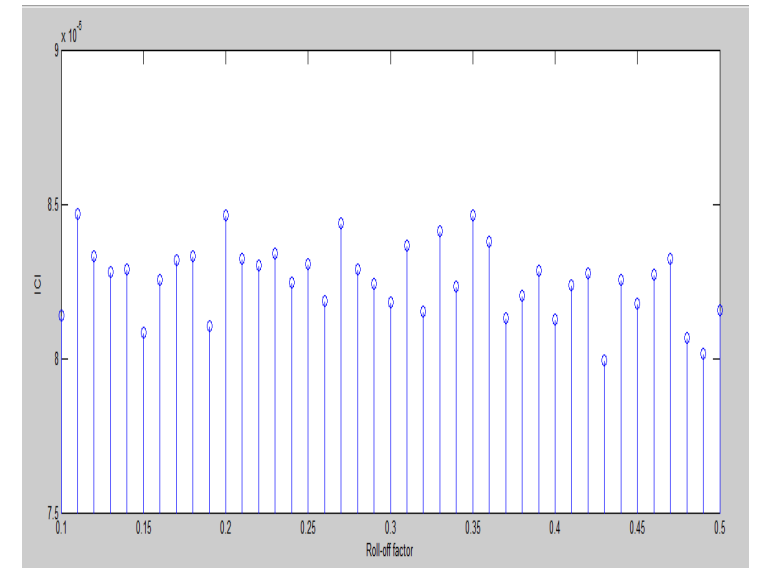

Fig. 8 .Plot of ICI vs. Roll off factor ( $\beta$ ) Optimised roll-off factor: 0.4300

\section{CONCLUSION}

In this paper, the authors have implemented the OFDM system with QPSK mapping scheme for Rayleigh faded multipath channel. It can be analyzed that with increase in frequency offset, Inter carrier interference power (ICI) and BER increases while Carrier to interference ratio decreases.ICI is responsible for degradation of OFDM system performance, so pulse shaping techniques are found to be good for reducing ICI in all existing ICI reduction techniques. Approximately $6 \mathrm{~dB}$ reduction in ICI and $6 \mathrm{~dB}$ increase in $\mathrm{C} / \mathrm{I}$ ratio at a value of 0.1 frequency offset is obtained after using RRC type of pulse shaping. Also, through adaptive pulse shaping, the authors have obtained optimum roll off factor of 0.43 at which minimum ICI value of $8 \times 10^{-5}$ approximately is obtained. Thus the reduction of ICI by using an RRC pulse shaping technique decreases the susceptibility of OFDM system with the Carrier frequency offset.

\section{REFERENCES}

[1] S.B. Weinstein and Paul M. Ebert, 'Data transmission by frequency division multiplexing using the Discrete Fourier Transform', IEEE Transactions on Communication Technologies, Vol. Com-19, No.5, October 1971

[2] Theodore S. Rappaport, A. Annamalai, R.M. Buehrer, and William H. Tranter, "Wireless Communications: Past events and future Perspectives", IEEE Communications Magzines, Page No: 148-161, May 2002

[3] J. Ahn, "Optimum finite length equalization for multicarrier transceivers", IEEE Trans. Communication, Vol.44, pp. 56 64, Jan 1996.

[4] R. Li and G. Stette, "Time -limited orthogonal multicarrier modulation schemes", IEEE Trans Commun., vol. 43, pp. 1269-1272, 1995.

[5] C. Muschallik, "Improving an OFDM reception using an adaptive Nyquist win- dowing", IEEE Trans. Consum. Electron. vol. 42, no. 3, pp. 259-269, 1996.

[6] Y. Zhao and S. G. Haggman, "Sensitivity to Doppler shift and carrier frequency errors in OFDM systems the consequences and solutions", IEEE 46th Vehicular Technology Conf., pp. 1564-1568, May 1996.

[7] Jean Armstrong, "Analysis of new and existing methods of reducing inter carrier interference due to carrier frequency offset in OFDM", IEEE Transaction on Communications, vol. 47, no.3, March 1999.

[8] Sanjay Mohanty and Susmita Das, "A Comparative Study of Pulse Shaping Functions for ICI Power Reduction in OFDM System", IEEE International Conference on Computer Communication and Informatics, 2008.

[9] V.N. Richard \& R Prasad, “OFDM for wireless Multimedia Communication”, Artech house publisher, London 2000.

\section{AUTHORS PROFILE}

Ashok R. Khedkar completed his B.E in Electronics and Telecommunication and M.Tech in Microwave from University of Pune in year 2003 and 2008 respectively. He is pursuing $\mathrm{PhD}$ from Sathyabama University, Chennai. His research areas are wireless Communication, Wave theory and Antennas. He is presently Assistant Professor in Cummins College of Engineering, Pune, Maharashtra, India

M.Sumathi received her Bachelor of Electronics and Communication Engineering Degree from Madras University and Master's Degree in Applied Electronics from Anna University, Chennai in 1992 and 2001 respectively. She completed her Doctoral degree in RF CMOS VLSI and current areas of interest include analog/Mixed Signal VLSI design, RF/Microwaves, Optical Communication Systems, Biomedical Image Processing and VLSI signal processing/testing. She is currently working as Professor in School of Electrical and Electronics Engineering, Sathyabama University, Chennai, India.

M. Murugan completed his BE from University of Madras in the year 1989, Later, he completed his M. E. and $\mathrm{Ph} . \mathrm{D}$ from University of Pune in the year 2001 and 2010 respectively. He has been actively involved in the field of technical education as academician, researcher, and administrator.He has over 70 papers published in National/International conferences/journals. Currently he is serving as Vice-principal and Professor of Department of Electronics and Communication Engineering of SRM's Valliammai Engineering College, Chennai, India. 